\title{
Analysis of Quality of Life among Caregivers of Patients with Thalassemia Major by Cross-Sectional Examination at a Tertiary Care Hospital
}

\author{
Authors: Unnithan VB ${ }^{1}$, Angane $A Y^{2}$, Ghorpade $\mathrm{GS}^{2}$, Kadam $\mathrm{KS}^{2}$ (mentor) \\ ${ }^{1}$ Intern, Seth GS Medical College and KEM Hospital, Mumbai, India \\ ${ }^{2}$ Department of Psychiatry, Seth GS Medical College and KEM Hospital, Mumbai, India \\ DOI: https://doi.org/10.26800/LV-142-supp5-39
}

\section{Introduction:}

Patients with thalassemia major are among the vulnerable subsets of population who need to be frequently admitted for transfusions. Thus, their caregivers are at an elevated risk of developing psychiatric comorbidities as they better understand the course of disease, prognosis and anticipate the impending burden. There is a lacuna in literature about our understanding of these caregivers' experience with psychiatric illness, quality of life, coping skills and the effect these have on them as well as their patient.

\begin{abstract}
Aim:
The study assessed the quality of life of thalassemia caregivers in various domains and their coping skills. The nature and prevalence of psychiatric morbidity which was experienced by caregivers was examined. Finally, the association of these and disease severity with the caregivers' sociodemographic variables was analysed.
\end{abstract}

\section{Materials \& Methods:}

At a tertiary care hospital, using Cochran's formula, a cross-sectional study with 100 caregivers, recruited by purposive sampling technique, was carried out over twelve months in the thalassemia day care centre run by the Department of Paediatrics. The caregivers were given a semi-structured proforma to collect sociodemographic data. General Health Questionnaire 12 (GHQ 12), WHO-Quality of Life-BREF (WHO-QOL BREF) and Coping Inventory for Stressful Situations 21 (CISS 21) scale was also administered. Contingency tables, Fischer's exact test and Mann Whitney $U$ test were used for statistical analysis.

\section{Results:}

Quality of life was independent of caregiver's sociodemographic variables. Significant difference was seen in psychological domain based on thalassemia severity. The authors observed $35 \%$ prevalence of psychiatric morbidity including depression and anxiety among caregivers. There was higher psychiatric morbidity in females. Coping skills were associated with employment $(p=0.009)$ and psychiatric morbidity $(p=0.001)$.

\section{Conclusion:}

Caregivers also experience psychological morbidity, necessitating therapy. Caregiver counselling will ensure better child care and improve treatment adherence.

Keywords: Caregiver, Coping Skills, Psychiatric Disorder, Quality of life, Thalassemia major 